\title{
Causes of Simultaneous Keystrokes in Children and Adults
}

\author{
Akiyo Kano and Janet C. Read \\ ChiCI Research Group, University of Central Lancashire, \\ Preston, PR2 2XD, England \\ \{Akano, jcread\}@uclan.ac.uk
}

\begin{abstract}
Simultaneously pressing two keys on a keyboard (Zero Time keystrokes) is a unique typing occurrence. To understand the cause of Zero Time keystrokes, typing data were collected from young children and undergraduate computing students. The results show that, in both students and children, the most frequent cause of Zero Time keystrokes were errors in aiming for the intended key, resulting in the intended and an adjacent key being pressed together. The second most frequent errors were Transposition Errors, which were errors in the ordering of the intended two letters.
\end{abstract}

Keywords: Typing error analysis, Simultaneous keystrokes, Zero Time keystrokes, Adjacent letter Errors, Transposition Error.

\section{Introduction}

In text input evaluation experiments, key loggers with timestamps are often used to record which keys were pressed and when they were pressed [1]. The logger records the time of each key pressed and allows researchers to see how long a participant took to complete a typing task and the time taken between each keystroke. Timing of keystrokes has been widely used in text input analysis for various purposes.

Using timestamps, Grudin [2] analysed behavioural differences between experts and novices ( 8 novices and 6 experts) and found that potentially, more than half of Insertion Errors examined (e.g. 'fox' for 'foix') involved two keys struck by the same finger. This led him to conclude that the error was in the execution, because it is assumed to be the result of faulty implementation of the keystroke. Salthouse [3] found that $54 \%$ of his Intrusion Errors were adjacent letters to the intended key. He used timestamps to investigate pauses in typing around Intrusion Errors and found that when an adjacent key is struck with the intended key, the timing between the two keystrokes was much shorter (less than 0.1 second) than average. He thus concluded that adjacent insertions occurred by a finger being incorrectly positioned between two keys, resulting in the two keys being pressed together.

Kano \& Read [1] defined the Zero Time keystroke as a keystroke where the time between it and the keystroke before it is recorded as 0.000 seconds by a key logger recording to the nearest 0.001 seconds. The NT/CT rules states that if the two keys had Zero Time interval, and they were Next-To or Close-To to each other, it can be 
assumed that the two keys were pressed with one finger and at the same time. They used this rule to decide whether the letter insertion occurred simultaneously with intended letters, or on its own, and were able to resolve ambiguities between insertion errors.

So far, an analysis of what error types could result in Zero Time keystrokes is yet to be found in literature. This paper focuses on Zero Time keystrokes to understand the causes of this unique typing anomaly.

\section{Experimental Design}

Two large studies using phrase copy typing were conducted to gather Zero Time keystrokes from undergraduate students and young children. Phrase copying was preferred to free text creation as it enables researchers to know what the participant intended to type [4].

A four day study was carried out with undergraduate first year students from the computer science department at the authors' university. Out of the 92 participants, there were 80 male and 12 female students, the majority (86) of whom were between the ages of 18-30, with 6 students in their 30s and 40s. All experiments were carried out in quiet computing labs, equipped with the same model of keyboard (HP KB0316). The investigator went into 7 practical classes of the same computing module and asked for volunteers to take part. There were less than 20 students per lab, and each had access to a PC. The procedure was explained to them fully before they started the task.

A second, five day study was also carried out in three schools in the local area, with children aged 6 to 10 years old. Of the 94 participants from 3 different schools, 58 were boys and 36 were girls. The children were from year 2 ( 6 to 7 year olds), year 3 ( 7 to 8 year olds) and year 5 (9 to 10 year olds) with the majority of the participants (79 children) from year 5 . The children had half an hour to carry out the typing in their schools' ICT suites, which could hold 10 to 15 children. The procedure was explained to them fully beforehand and they worked on individual computers. As the child study was carried out in three schools, the equipment varied a little, with different makes and models of keyboards (Labtec Y-SAM64, Dell SK-8115 and HP KB0136) However, they were all full sized, white letters on black keyboards with British keyboard layout.

In both studies a data-collecting tool GetItAll [1] was used to collect personal information about the participants, present the phrases and collect the typing data. Although adjustments were made between the students and the children in gathering personal information, the copy typing task itself was kept the same so the data remained comparable. Each participant ran an individual copy of GetItAll on his or her PC, and had 10 randomly selected phrases from the CPSet [5] to copy. The phrases were shown one at a time in font style Verdana at size point 14, with a space for the participant to copy the phrase underneath. The data collector logged what phrases were shown, each key that was pressed, recorded its timestamp and the time between the keystrokes. 
The key logs were analysed for any keystrokes that were Zero Time. If the Zero Time key press did not cause an error, e.g. the two keys pressed simultaneously were the correct letters in the correct order, then it was classified as No Error.

For those Zero Time keystrokes that caused an error, these were divided according to what letters were involved. If the two letters pressed simultaneously were adjacent to each other, then they were categorised as NT-Mu or CT-Mu. Next To Error Multiple Key Presses (NT-Mu) refers to when a key directly next to the intended key is pressed along with the intended key (e.g. 'ourt' for 'out'). Close To error Multiple key presses (CT-Mu) refers to when one or more keys 'close to' but not next to the intended key are pressed together with the intended key (e.g. 'onl7y6' for 'only').

For the remaining Zero Time keystrokes, which caused an error but did not involve letters that were Next-To or Close-To each other, they were further divided into Transposition Errors (TE) or not. Transposition Errors (TE) refers to when consecutive letters are switched, such as 'tiem' for 'time'.

\section{Experimental Results}

23668 keystrokes were collected from the student study, of which 72 were Zero Time keystrokes. 22224 keystrokes were collected from the child study, of which 54 were Zero Time keystrokes. Figure 1 shows the number of Zero Time keystrokes found in each category for both students (shown in bold) and children (shown in italics) in percentages of the total Zero Time keystrokes found.

There were 6 Zero Time keystrokes in the student study and 12 in the child study that resulted in No Error. These No Errors occurred when the two letters pressed

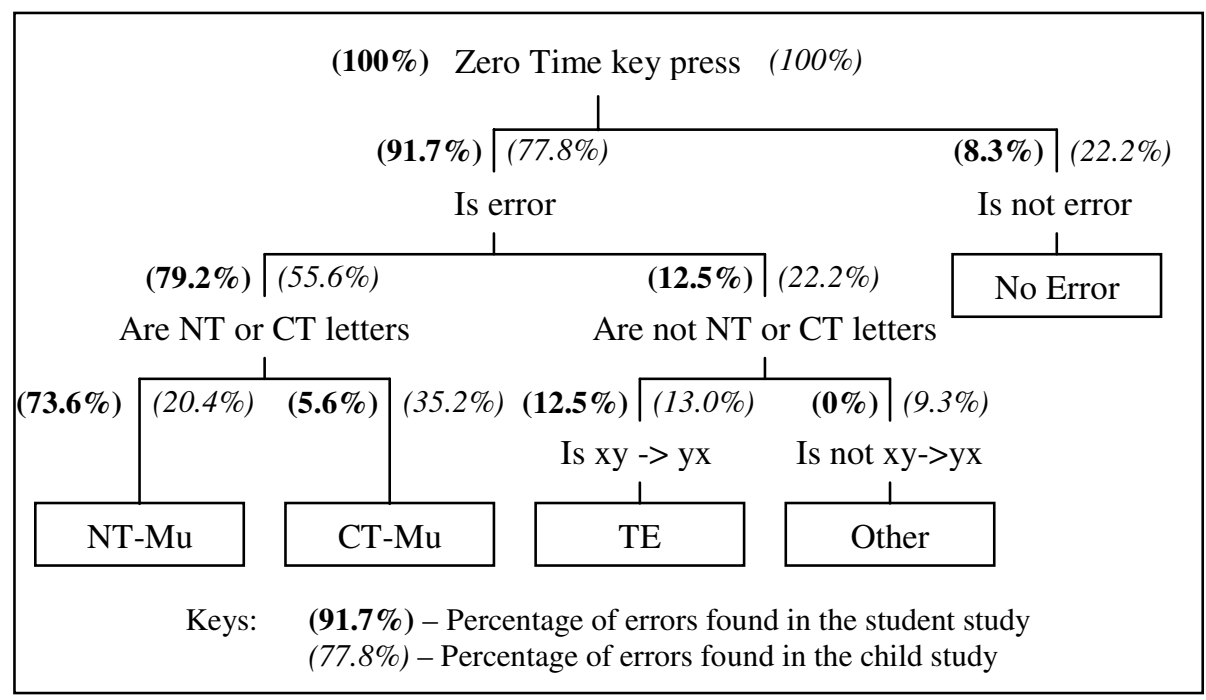

Fig. 1. Percentage of Zero Time Keystrokes Found in Each Category of Typing Errors for Students and Children 
together were the correct letters, and despite the Zero Time, were recorded by the keyboard to have been pressed in the right order.

Interestingly, the students had made considerably more NT-Mu errors (73.6\%) than CT-Mu errors (5.6\%), while children made higher number of CT-Mu (35.2\%) than NT-Mu (20.4\%). One possible explanation is that some children resting their hands below the keyboard between keystrokes, and to reach any key, their hands must move vertically first, whereas adults tend to hover their hands over the keyboard and move more sideways. It is hard to conclude a cause for this observation, without a detailed study on the starting position of hands in between keystrokes in both children and adults.

Of all Zero Time keystrokes found, $91.7 \%$ in student study and $77.8 \%$ were either NT-Mu or CT-Mu. This suggests that the majority of Zero Time keystrokes are caused by pressing two adjacent keys when only one was intended. The next major group of errors were the Transposition Error at $12.5 \%$ for students and $13.0 \%$ children.

\section{Conclusion}

Zero Time keystrokes were collected from young children and undergraduate computing students and analysed. The results show that the in both children and students, the vast majority (average of $84 \%$ ) of Zero Time keystrokes were due to misaim of the finger, resulting in the one finger pressing two keys together.

With respect to ratio of the different error types found, there were some interesting variations between the student and children study, such as the children's NT-Mu and $\mathrm{CT}-\mathrm{Mu}$ ratio that were reversed in the student study. These variations require further investigation as to the causes.

\section{References}

1. Kano, A., Read, J.C.: Text Input Error Categorisation: Solving Character Level Insertion Ambiguities Using Zero Time Analysis. In: HCI 2009. ACM, Cambridge (2009)

2. Grudin, J.T.: Error Patterns in Novice and Skilled Transcription Typing. In: Cooper, W.E. (ed.) Cognitive Aspects of Skilled Typing, pp. 121-139. Springer, New York (1983)

3. Salthouse, T.A.: Perceptual, Cognitive, and Motoric Aspects of Transcription Typing. Psychological Bulletin 99(3), 303-319 (1986)

4. MacKenzie, I.S., Soukoreff, R.W.: Phrase Sets for Evaluating Text Entry Techniques. In: Extended Abstracts of the ACM Conference on Human Factors in Computing Systems CHI 2003. ACM Press, New York (2003)

5. Kano, A., Read, J.C., Dix, A.: Children's Phrase Set for Text Input Method Evaluations. In: Proceedings of the Fourth Nordic Conference on Human-Computer Interaction - NordiCHI. ACM Press, Oslo (2006) 